\title{
Observation of a Chiral State in a Microwave Cavity
}

\author{
C. Dembowski, ${ }^{1}$ B. Dietz, ${ }^{1}$ H.-D. Gräf, ${ }^{1}$ H.L. Harney,${ }^{2}$ A. Heine,${ }^{1}$ W. D. Heiss,${ }^{3}$ and A. Richter, , \\ ${ }^{1}$ Institut für Kernphysik, Technische Universität Darmstadt, D-64289 Darmstadt, Germany \\ ${ }^{2}$ Max-Planck-Institut für Kernphysik, D-69029 Heidelberg, Germany \\ ${ }^{3}$ Department of Physics, University of Stellenbosch, 7602 Matieland, South Africa
}

(Dated: November 8, 2018)

\begin{abstract}
A microwave experiment has been realized to measure the phase difference of the oscillating electric field at two points inside the cavity. The technique has been applied to a dissipative resonator which exhibits a singularity - called exceptional point - in its eigenvalue and eigenvector spectrum. At the singularity, two modes coalesce with a phase difference of $\pi / 2$. We conclude that the state excited at the singularity has a definitiv chirality.

PACS numbers: 05.45.Mt, 41.20.Jb, 03.65.Vf, 02.30.-f
\end{abstract}

Recently a surprising phenomenon occurring in systems described by non-hermitian Hamiltonians has received considerable attention: the coalescence of two eigenmodes. If the system depends on some interaction parameter $\lambda$, the value $\lambda_{\mathrm{EP}}$ at which the coalescence occurs is called an exceptional point (EP) 1]. At an EP, the eigenvalues and eigenvectors show branch point singularities [1, 2, 3, 4, 5] as functions of $\lambda$. This stands in sharp contrast to two-fold degeneracies, where no singularity but rather a diabolic point [ [6] occurs. EPs have been observed in laser induced ionization of atoms [7], acoustical systems [8], microwave cavities [9, 10], certain absorptive media 11, and in "crystals of light" 12]. The broad variety of systems showing EPs indicates that their occurrence is generic which is discussed further in 3, 13. So far EPs have been observed in decaying systems described by a complex symmetric effective Hamiltonian [14]. While the theoretical and experimental articles cited above discuss the properties of systems in the vicinity of an EP, a recent theoretical work 15] investigates the complex symmetric Hamiltonian of a two-level system at the EP. The eigenfunction at the EP turns out to be

$$
\left|\psi_{\mathrm{EP}}\right\rangle \propto|1\rangle \pm i|2\rangle
$$

for any choice of the basis states $|1\rangle$ and $|2\rangle$. This is a chiral state: in quantum, acoustical and electromagnetic systems the two orthogonal basis states oscillate in time; if they are superimposed according to Eq. (1) where they follow each other with a time lag of a quarter period - the result is rotating either clockwise or counter-clockwise. This is in analogy with the generation of circularly polarized light being a superposition of two orthogonal linearly polarized waves phase shifted by $\pi / 2$. The sign of the chirality is defined via the direction of time and in the experiment the positive direction of time is given by the decay of the eigenstates. The first observation of such a chiral state in a microwave cavity experiment is the gist of the present paper.

The high precision of such an experiment makes it a prime choice to observe the chirality of $\left|\psi_{\mathrm{EP}}\right\rangle$, yet measur- ing a phase difference of $\pm \pi / 2$ between two superimposed modes is an unusual and demanding experimental task. A setup had to be designed that allows to excite the two modes with an adjustable frequency difference $f_{1}-f_{2}$, and in the case of $f_{1}=f_{2}$ with an adjustable phase difference between them. The cavity is composed of two almost identical semi-circular parts, see Fig. 11 They are coupled by a slit of variable width $s$. In order to reach $f_{1}=f_{2}$ one needs a second tunable parameter, namely the position $\delta$ of a teflon semi-circular stub in part 1 of the cavity on Fig. 10 These two parameters make up the

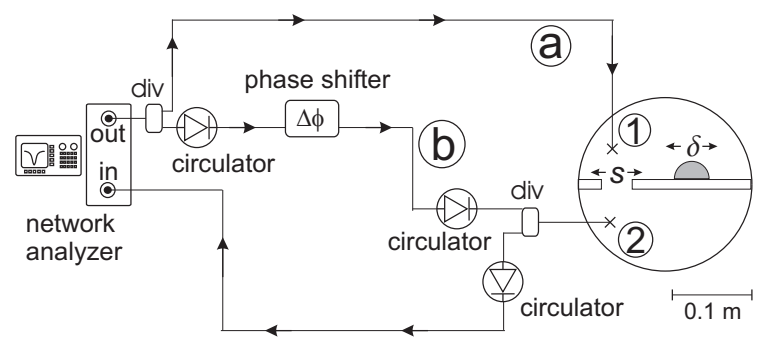

FIG. 1: Experimental setup to measure the phase shift between two different positions in the cavity. The geometry of the resonator can be changed by adjusting the widths $s$ of a slit between the two halves (labeled (1) and (2), resp.) and the position $\delta$ of a teflon stub. Microwave power is coupled into the resonator via path (a) and antenna 1 into part (1) and, with a tunable phase shift $\Delta \phi$, via antenna 2 into part (2), where it is also picked up.

interaction parameter $\lambda(s, \delta)$. The two eigenmodes coalesce at a critical value $\lambda_{\mathrm{EP}}=\left(s^{\mathrm{EP}}, \delta^{\mathrm{EP}}\right)$. In the sequel we chose $\lambda$ to be close enough to $\lambda_{\mathrm{EP}}$ so as to consider the cavity a two-state-system. This is possible when the distance between the two coalescing eigenvalues is much smaller than the distance to any third one. As in [10], the EP has been found by looking at the behavior of the real and imaginary parts of the eigenvalues, i.e. the resonance frequencies $f_{1}, f_{2}$ and widths $\Gamma_{1}, \Gamma_{2}$. For weak coupling, i.e. $s<s^{\mathrm{EP}}$, one observes a crossing of $f_{1}, f_{2}$ and an avoided crossing of $\Gamma_{1}, \Gamma_{2}$ when one sweeps $\delta$. For strong coupling, i.e. $s>s^{\mathrm{EP}}$, an avoided crossing of 
$f_{1}, f_{2}$ and a crossing of $\Gamma_{1}, \Gamma_{2}$ takes place [2, 10, 16]. It is only at the EP that one expects the real and imaginary parts of the eigenvalues to be equal. In addition to the eigenvalues, the eigenfunctions were studied by mapping the distributions of the electric field. When the EP is encircled in the space of $\lambda$, one of the eigenvectors undergoes a change of sign [10], i.e. it picks up a so-called "geometric phase" [17]. Which eigenvector changes its sign is defined by the only freedom left: the orientation of the closed loop around the EP. This is a first fingerprint of the definite chirality of the EP.

For $s<s^{\mathrm{EP}}$ the two modes discussed in the following are each localized in one of the semi-circular parts of the cavity - as the measured field distributions show (cf. insets of Fig. 2). We consider these localized modes as the basis configurations $|1\rangle$ and $|2\rangle$ of the two-state system. They can be excited separately even if $f_{1}=f_{2}$ by

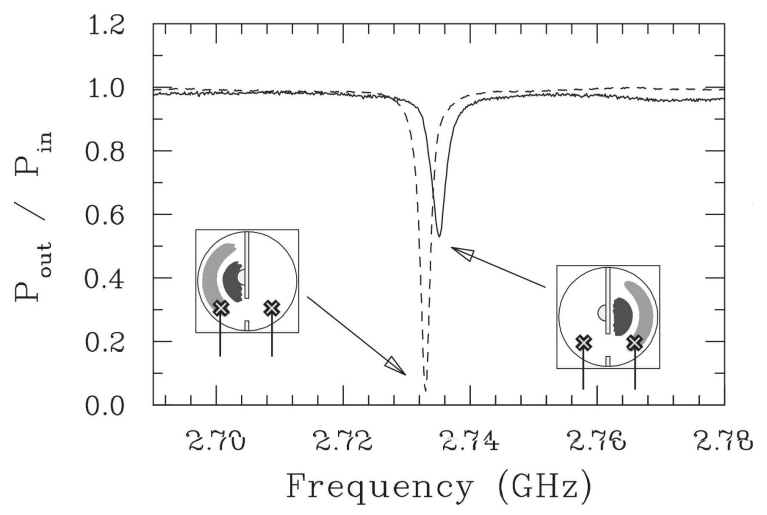

FIG. 2: Reflection spectra, i.e. the outcoupled power over the incoupled one as a function of frequency, taken with two different antennas in mirrored positions marked by crosses with attached lines. The full (dashed) line is taken with the antenna in the right (left) semicircular part of the cavity. In the frequency interval shown lie two eigenmodes of the resonator with field distributions plotted as insets, however, in each spectrum only a single resonance is visible since each antenna couples to only one of the two eigenmodes.

appropriately positioned dipole antennas 18 which are located at mirror positions in the cavity. The coupling between the antennas and the resonator is proportional to the square of the electric field at the locations of the antennas [18, 19. If the electric field is approximately zero at the location of a given antenna the mode cannot be excited. In Fig. 2 the reflection spectra for two different antennas are shown for $s=38 \mathrm{~mm}$. The parameter $\delta$ is tuned so that that $f_{1}$ is slightly different from $f_{2}$. Still each spectrum shows only a single resonance - not a doublet. It follows that each antenna indeed excites only one basis configuration.

In the two-state-regime, the eigenstates $\left|\psi_{k}\right\rangle$ of the cavity are expanded according to

$$
\left|\psi_{k}\right\rangle=a_{k 1}|1\rangle+a_{k 2}|2\rangle,
$$

however, for sufficiently small $s$, the eigenmodes $\left|\psi_{k}\right\rangle$ approach the localized modes $|1\rangle$, and $|2\rangle$ as can be seen in Fig. 2] The relative phase of the amplitudes $a_{k 1}$ and $a_{k 2}$ of $\left|\psi_{1}\right\rangle$ and $\left|\psi_{2}\right\rangle$ has been measured by the following technique, applied - to the best of our knowledge - for the first time in microwave cavities. A microwave source, an HP8510C vectorial network analyzer is emitting continuous rf-radiation with a fixed frequency, split by a power divider (Narda 4313-2) and fed into the cavity via two paths labeled (a) and (b) in Fig. 10 The phase of the signal travelling through (b) can be shifted by $\Delta \phi$ using a Narda 3752 phaseshifter. Several circulators 20] are used to suppress reflections along path (b). The amplitude $S$ of the signal outcoupled by antenna 2 is a superposition of two coherent waves with different phases, i.e.

$$
S \propto t+r e^{i \Delta \phi} .
$$

Here, $t$ and $r$ denote the transmission and reflection coefficients of the cavity. For further analysis, two cases have to be distinguished: First, for $s<s^{\mathrm{EP}}, \delta$ is tuned in such a way that the frequencies of the two eigenstates $\left|\psi_{1}\right\rangle$ and $\left|\psi_{2}\right\rangle$ are the same and both are excited in resonance by the two signals coupled to the cavity. Second, for $s=s^{\mathrm{EP}}$, the only state present, $\left|\psi_{\mathrm{EP}}\right\rangle$, is excited by both signals.

In both cases, $t$ and $r$ are known from scattering theory (see e.g. [21, 22, 23]) and the dependence of the signal received by the network analyzer from $\Delta \phi$ is given by

$$
|S(\Delta \phi)|^{2} \propto C+\cos ^{2}\left(\frac{\Delta \phi-\phi_{0}-\tilde{\phi}}{2}\right) .
$$

Here, the constant $C \geq 0$ determines the contrast of the pattern. When $C$ is large, the contrast is small, which occurs whenever the basis states contribute very unevenly to the eigenmodes. The angle $\phi_{0}$, i.e. the phase difference between the oscillating fields at the position of the antennas, is given by the relative phases of the expansion coefficients in (2), i.e.

$$
\phi_{0}=\arg \left(a_{12} / a_{11}\right)
$$

for an isolated eigenmode and

$$
\phi_{0}=\arg \left(\frac{a_{12}+a_{22}}{a_{21}}\right)
$$

for the superposition of two eigenmodes. If Eq. (1) holds true we expect $\phi_{0}= \pm \pi / 2$ for $\left|\psi_{\mathrm{EP}}\right\rangle$, proving the chirality of $\left|\psi_{\mathrm{EP}}\right\rangle$.

The angle $\tilde{\phi}$ in Eq. (4) is due to the remaining length difference of the paths (a) and (b) and the fact that the antennas (1) and (2) may cause additional and different phase shifts. It has to be determined by analyzing an eigenstate with known $\phi_{0}$. One easily finds [19] modes with $\phi_{0}=0$ or $\pi$. Figure 3 a shows $|S(\Delta \phi)|^{2}$ for a mode 


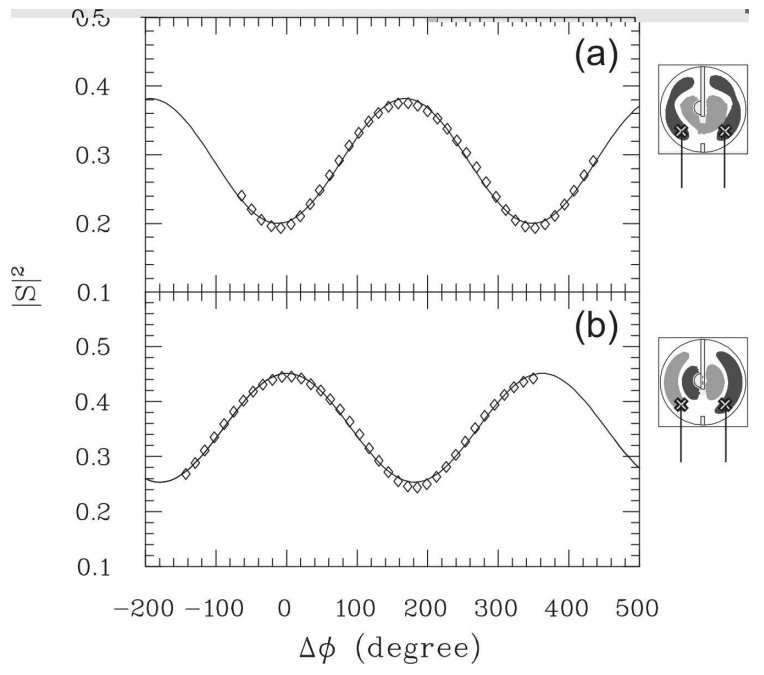

FIG. 3: The square of the amplitude of the signal outcoupled by antenna $2,|S|^{2}$, as a function of the phase shift $\Delta \phi$. In (a) the phase difference $\phi_{0}$ of the oscillating electric field between the antennas (marked as crosses with attached lines) is 0 for the measured field distribution shown, while in (b) it is $\pi$. In both cases the minimum of $|S(\Delta \phi)|^{2}$ corresponds to $\phi_{0}$.

with $\phi_{0}=0$, i.e. the electric field at the position of the two antennas oscillates in phase, cf. Fig. [3k, where the antennas are sketched as crosses with attached lines. By fitting Eq. (4) to the measured $|S(\Delta \phi)|^{2}$ we find $\tilde{\phi} \approx \pi$, which is expected from measurements of the reflection coefficients 24] of the antennas which shift the phase of an emitted wave by $\pi$. In Fig. Bb we show $|S(\Delta \phi)|^{2}$ for a mode with $\phi_{0}=\pi$, as can be seen from the measured field distribution. The data agrees again well with Eq. (4).

Having verified that this setup allows to measure the phase shift between different points of a field distribution, we turn to the investigation of $\left|\psi_{\mathrm{EP}}\right\rangle$. To reach the EP the slit is at first set to $s=3 \mathrm{~mm}$, i.e. $s<s^{\mathrm{EP}}$. The teflon semi-circle can be moved from the outside, i.e. while microwave power is coupled into the cavity and therefore $\delta$ can be tuned such that $f_{1}=f_{2}$. Then the pattern $|S(\Delta \phi)|^{2}$ exhibits only a weak contrast (see the upper part of Fig. (4). This occurs because $\left|a_{12}\right|$ is very small compared to $\left|a_{11}\right|$ and $\left|a_{22}\right|$ is large compared to $\left|a_{21}\right|$ for such a weak coupling. Nevertheless, $\phi_{0}$ can be obtained by fitting Eq. (4) to the measured data. It is close to $\pi$ for $s<28 \mathrm{~mm}$, cf. Fig. [5] which agrees with general arguments given below.

Secondly we set $\lambda \approx \lambda_{\mathrm{EP}}$ so that $\left|\psi_{\mathrm{EP}}\right\rangle$ is the only mode present (lower part of Fig. (4). The contrast of the pattern $|S(\Delta \phi)|^{2}$ is high because the expansion coefficients of $\left|\psi_{\mathrm{EP}}\right\rangle$ have the same absolute value. For $s=46 \mathrm{~mm}$ one obtains $\phi_{0}=\pi / 2 \pm 0.05$, cf. Fig. 5 This is the phase predicted in Eq. (II) - constituting the first experimental observation of a mode, were the electric field between two points oscillates with a phase difference of $\pi / 2$. As pointed out, this is a chiral mode rotating clockwise in

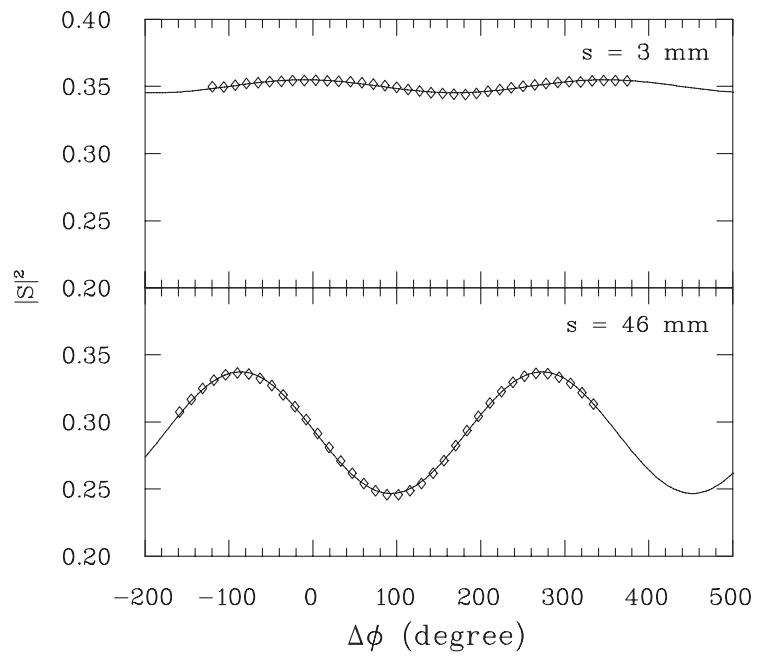

FIG. 4: The square of the amplitude of the signal outcoupled by antenna $2,|S|^{2}$, as a function of the phase shift $\Delta \phi$ for weak coupling $(s=3 \mathrm{~mm})$ and at the $\operatorname{EP}(s=46 \mathrm{~mm})$. As in Fig. 3 the minimum of $|S(\Delta \phi)|^{2}$ corresponds to the phase difference of the oscillating electric field between the antennas.

the basis frame spanned by $|1\rangle$ and $|2\rangle$.

Figure $\left[5\right.$ shows the behavior of $\phi_{0}$ from small to large slit openings. For each value of $s,|S(\Delta \phi)|^{2}$ has been taken as displayed on Fig. [4 by sweeping $\Delta \phi$ over $\approx 400^{\circ}$. The error of $\phi_{0}$ is a systematic error caused by varying bending radii of the coaxial microwave cables and the corresponding variation of the length of the wave paths during the measurement. The surface properties of the connections between the cables and the antennas which cannot be exactly controlled - are another source of error.

For $43 \mathrm{~mm}<s<48 \mathrm{~mm}$ a crossing of the resonance frequencies and widths is observed and we conclude that this is the precision with which the present experiment yields $s^{\mathrm{EP}}$. This does not contradict the theory [2]- the experiment simply cannot resolve the tiny avoided frequency or widths crossings that occur for $s$ very near to $s^{\mathrm{EP}}$.

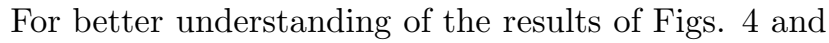
[5 let us recapitulate the two-state model of [15]. The Hamiltonian

$$
H=\left(\begin{array}{cc}
E_{1}-i \gamma_{1} & H_{12} \\
H_{12} & E_{2}-i \gamma_{2}
\end{array}\right)
$$

is complex symmetric. All its entries are functions of the geometric parameters $\lambda$. An EP occurs whenever the eigenvalues of $H$ coincide and $H_{12} \neq 0$. The sum of the relative amplitudes occurring in Eq. (6) is

$$
\frac{a_{12}}{a_{11}}+\frac{a_{22}}{a_{21}}=\frac{\Delta E-i \Delta \gamma}{H_{12}},
$$

where $\Delta E=E_{1}-E_{2}$ and $\Delta \gamma=\gamma_{1}-\gamma_{2}$. At the EP, 


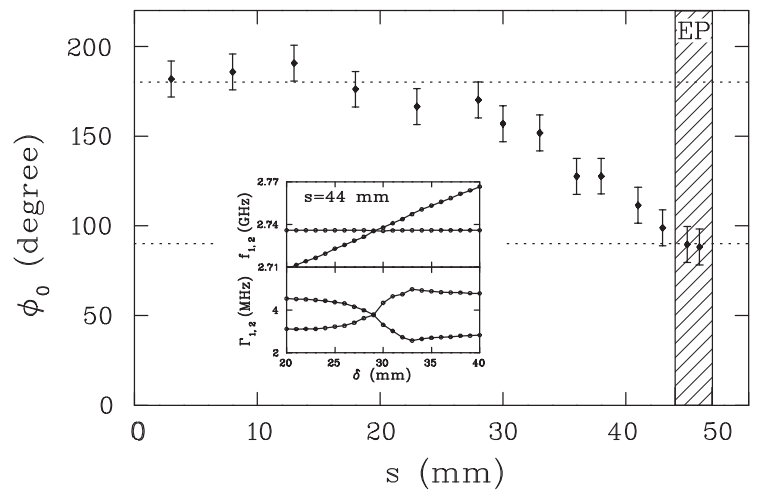

FIG. 5: The phase difference $\phi_{0}$ of the oscillating electric field between the antennas for different couplings $3 \mathrm{~mm} \leq$ $s \leq s^{\mathrm{EP}}$. The EP is reached for $44 \mathrm{~mm} \leq s \leq 47 \mathrm{~mm}$ (shaded area), where we find, within the resolution of the experiment, $f_{1} \approx f_{2}$ and $\Gamma_{1} \approx \Gamma_{2}$. The phase difference at the EP is $\phi_{0}=(90 \pm 3)^{\circ}$, indicating the chirality of $\left|\psi_{\mathrm{EP}}\right\rangle$. As an inset we show the crossing of the resonance frequencies and widths for $s=44 \mathrm{~mm} \approx s^{E P}$

Eq. (8) becomes

$$
\frac{a_{12}}{a_{11}}+\frac{a_{22}}{a_{21}}= \pm 2 i .
$$

The argument of the l.h.s of Eq. (9) coincides with the r.h.s of Eq. (6) if $a_{11}=a_{21}$ as is the case in the experiment. For a weak coupling the r.h.s. of Eq. (8) is large because $H_{12}$ approaches zero linearly with $s$. As we enforce equality of the real parts of the eigenvalues of $H$, a particular weak coupling limit is specified. It implies $\Delta E=0$. The finding $\phi_{0} \approx \pi$ in Fig. 5 means that $H_{12}$ tends towards zero along the imaginary axis. This describes just the coupling within a purely absorptive system.

In summary, we have performed a microwave cavity experiment to measure the phase difference $\phi_{0}$ between two points of a field distribution inside the resonator. The technique has been applied to the superposition of two eigenmodes on a path to an EP. At the EP, the modes coalesce into the single, non-localized mode $\left|\psi_{\mathrm{EP}}\right\rangle=|1\rangle+i|2\rangle$, i.e. $\phi_{0}=\pi / 2$. The phase $\phi_{0}$ is determined from the pattern $|S(\Delta \phi)|^{2}$ of interference between the amplitude for transmission through the cavity and a wave with the arbitrary phase shift $\Delta \phi$. To our knowledge, it represents the first experimental observation of a mode in a microwave resonator, where the electric field oscillates with a phase difference of $90^{\circ}$ at two different points.

The decay of the eigenmodes is essential for the EP to occur. Therefore the experiment has been performed at room temperature rather than under conditions of superconductivity [18]. The irreversibility of the decay causes $\left|\psi_{\mathrm{EP}}\right\rangle$ to change under the operation of time reversal. This should not be confused with fundamental time reversal symmetry breaking observed e.g. in the system of the neutral Kaons 25]. There the Hamiltonian of the decaying two-state system is not complex symmetric. Similarly, the chirality of $\left|\psi_{\mathrm{EP}}\right\rangle$, i.e. the fact that $|1\rangle+i|2\rangle$ is rotating clockwise, is not due to a parity violating interaction as in $\beta$-decay 26]. It rather is a property of the EP that we have investigated. The set of EPs that one can find in the resonator is expected to exhibit both chiralities [2]. In this sense the present chirality closely resembles the chirality of molecules [27], underlining the importance and the generic aspects of EPs even further.

We are particularly grateful to T.H. Seligman for discussing the results with us. C.D., B.D., and A.H. thank CONACyT for financial support during the workshop on Chaos in few and many body problems at CIC. This work has been supported by the DFG under contract number Ri 242/16-3.

* Electronic address: richter@ikp.tu-darmstadt.de

[1] T. Kato, Perturbation theory of linear operators (Springer, Berlin, 1966).

[2] W.D. Heiss and A.L. Sannino, J. Phys. A 23, 1167 (1990); Phys. Rev. A 43, 4159 (1991).

[3] M.V. Berry and D.H.J. O'Dell, J. Phys. A 31, 2093 (1998).

[4] E. Hernández, A Jáuregui, and A Mondragón, J. Phys. A 33, 4507 (2000).

[5] I. Rotter, Phys. Rev. E 65, 26217 (2002). We disagree with this publication in that it holds exceptional points and diabolic points to be one and the same type of singularity.

[6] M.V. Berry and M. Wilkinson, Proc. R. Soc. Lond. A 392, 15 (1984).

[7] O. Latinne, N. J. Kylstra, M. Dörr, J. Purvis, M. TeraoDunseath, C. J. Joachain, P. G. Burke, and C. J. Noble, Phys. Rev. Lett. 74, 46 (1995).

[8] A.L. Shuvalov and N.H. Scott, Acta Mech. 140, 1 (2000).

[9] P.v. Brentano and M. Philipp, Phys. Lett. B 454, 171 (1999); M. Philipp, P. von Brentano, G. Pascovici, and A. Richter, Phys. Rev. E 62, 1922 (2000).

[10] C. Dembowski, H.-D. Gräf, H. L. Harney, A. Heine, W. D. Heiss, H. Rehfeld, and A. Richter, Phys. Rev. Lett. 86, 787 (2001).

[11] S. Pancharatnam, Proc. Ind. Acad. Sci. XLII, 86 (1955).

[12] M.K. Oberthaler, R. Abfalterer, S. Bernet, J. Schmiedmayer, and A. Zeilinger, Phys. Rev. Lett. 77, 4980 (1996).

[13] M.V. Berry, Curr. Sci. 67, 220 (1994).

[14] V. Weisskopf and E. Wigner, Z. Physik 63, 54 (1930).

[15] W.D. Heiss and H.L. Harney, Eur. Phys. J. D 17, 149 (2001).

[16] W.D. Heiss, Eur. Phys. J. D 7, 1 (1999); Phys. Rev. E 61, 929 (2000).

[17] M.V. Berry, Quantum Chaos, ed. by G. Casati (Plenum, London, 1985); Proc. R. Soc. Lond. A 392, 45 (1983).

[18] A. Richter, in Emerging Applications of Number Theory, The IMA Volumes in Mathematics and its Applications, Vol. 109, edited by D.A. Hejhal, J. Friedman, M.C. Gutzwiller, and A.M. Odlyzko, p. 479 (Springer, 
New York, 1999)

[19] J. Stein and H.J. Stöckmann, Phys. Rev. Lett. 68, 2867 (1992).

[20] J. Helszajn, Principles of Microwave ferrite engineering, (Wiley, New York, 1975).

[21] A.M. Lane and R.G. Thomas, Rev. Mod. Phys. 30, 257 (1958).

[22] C. Mahaux and H.A. Weidenmüller, Shell-Model Approach to Nuclear Reactions, North-Holland, Amsterdam 1969.

[23] H. Alt, H.-D. Gräf, H.L. Harney, R. Hofferbert, H. Lengeler, A. Richter, P. Schardt, and H.A. Weidenmüller, Phys. Rev. Lett. 74, 62 (1995).
[24] H. Padamsee and J. Knobloch and T. Hays, RF Superconductivity for Accelerators (Wiley, New York, 1998).

[25] CPLEAR Collaboration, Phys. Lett. B444, 43 (1998).

[26] C.S. Wu, E. Ambler, R. W. Hayward, D. D. Hoppes, and R. P. Hudson, Phys. Rev. 105, 1413 (1957); S.G. Crane, S. J. Brice, A. Goldschmidt, R. Guckert, A. Hime, J. J. Kitten, D. J. Vieira, and X. Zhao, Phys. Rev. Lett. 86, 2967 (2001).

[27] L. Pasteur, Comptes Rend. Hebd. Séances Acad. Sci. 26, 535 (1848); N. Böwering T. Lischke, B. Schmidtke, N. Müller, T. Khalil, and U. Heinzmannet, Phys. Rev. Lett. 86, 1187 (2001). 